\title{
MODIS Radiometric Calibration Program, Methods and Results
}

\author{
Xiaoxiong (Jack) Xiong ${ }^{1}$, Bruce Guenther ${ }^{2}$ \\ Amit Angal $^{3}$, William Barnes ${ }^{2}$, Vincent Salomonson ${ }^{4}$, Junqiang Sun ${ }^{5}$, and Brian Wenny ${ }^{5}$ \\ ${ }^{1}$ Sciences and Exploration Directorate, NASA/GSFC, Greenbelt, MD 20771, USA \\ ${ }^{2}$ University of Maryland, Baltimore County, 1000 Hilltop Circle, Baltimore, MD 21250, USA \\ ${ }^{3}$ Sciences and System Applications Inc., 10210 Greenbelt Road, Lanham, MD 20706, USA \\ ${ }^{4}$ University of Utah, Salt Lake City, UT 84112, USA \\ ${ }^{5}$ Sigma Space Corporation, 4801 Forbes Boulevard, Lanham, MD 20706, USA
}

\begin{abstract}
As a key instrument for NASA's Earth Observing System (EOS), the Moderate Resolution Imaging Spectroradiometer (MODIS) has made significant contributions to the remote sensing community with its unprecedented amount of data products continuously generated from its observations and freely distributed to users worldwide. MODIS observations, covering spectral regions from visible (VIS) to long-wave infrared (LWIR), have enabled a broad range of research activities and applications for studies of the earth's interactive system of land, oceans, and atmosphere. In addition to extensive pre-launch measurements, developed to characterize sensor performance, MODIS carries a set of on-board calibrators (OBC) that can be used to track onorbit changes of various sensor characteristics. Most importantly, dedicated and continuous calibration efforts have been made to maintain sensor data quality. This paper provides an overview of the MODIS calibration program, on-orbit calibration activities, methods, and performance. Key calibration results and lessons learned from the MODIS calibration effort are also presented in this paper.
\end{abstract}

\section{Index Terms - MODIS, Terra, Aqua, Calibration}

\section{INTRODUCTION}

Since launch, Terra and Aqua MODIS have successfully operated for more than 12 and 10 years, respectively. MODIS observations are made in 36 spectral bands, covering spectral regions from visible (VIS) to long-wave infrared (LWIR), and at $250 \mathrm{~m}$ (2 bands), $500 \mathrm{~m}$ (5 bands), and $1 \mathrm{~km}$ (29 bands) spatial resolutions at nadir. Various data products, covering a broad range of research activities and applications, have been continuously generated from MODIS observations and freely distributed to users worldwide and, as a result, significantly contributed to the earth remote sensing community [1]. In order to maintain sensor on-orbit calibration and data product quality, MODIS was designed and built with a full set of on-board calibrators (OBC), which include a solar diffuser (SD), a solar diffuser stability monitor (SDSM), a blackbody (BB), and a spectroradiometric calibration assembly (SRCA). The SD and SDSM are used together for the reflective solar bands (RSB) calibration and the $\mathrm{BB}$ for the thermal emissive bands (TEB) calibration. The primary use of the SRCA is to characterize the sensor spatial and spectral performance [2].

In addition to the OBC, regularly scheduled lunar observations and long-term trending of sensor responses over selected ground targets at different angle of incidence (AOI) to the sensor (scan mirror) are used in support of MODIS on-orbit calibration and characterization. This paper provides an overview of the MODIS radiometric calibration program and on-orbit calibration activities. It describes various methods currently implemented to calibrate MODIS RSB and TEB, and summarizes sensor on-orbit performance and key calibration results. Also discussed in this paper are challenging issues and special efforts made to assure mission success and long-term calibration quality.

\section{CALIBRATION PROGRAM AND ACTIVITIES}

The MODIS pre-launch calibration and characterization program was initially established by the sensor builder, Hughes, Santa Barbara Research Center (SBRC) of Goleta, CA. The formal input of the science community was incorporated in test program improvements through the implementation of a MODIS Calibration Group, the predecessor of the MODIS Characterization Support Team (MCST), within the MODIS Science Team. The calibration plan was further validated through an EOS peer-review process by the Calibration and Validation Working Group (CVWG), which included calibration experts from Europe, Asia, as well as North America. A key element for the successful accomplishment of the MODIS test plans involved the support of a core sensor builder team interacting with the Science Team and representing the performance community interests within the sensor builder 
community. Extensive pre-launch calibration and activities were performed by the instrument vendor to evaluate and determine sensor radiometric, spatial, and spectral performance at ambient and thermal vacuum environment. Measurements were also made to characterize sensor polarization sensitivity and response versus scan angle (RVS). MCST, working closely with the instrument vendor, has provided comprehensive calibration data analyses to support evaluation of sensor pre-launch performance.

In addition to supporting sensor pre-launch calibration and characterization test data analysis, and the Level 1B (L1B) algorithm design and implementation, MCST has undertaken the responsibility for sensor on-orbit operation, calibration planning and execution. Sensor performance and calibration results are regularly presented at the MODIS Science Team meetings and special calibration workshops, and made available to the public. The MCST effort has been supported by the MODIS Program and Project and maintained throughout different calibration phases. As part of the MODIS calibration program, a dedicated MODIS sensor Working Group (MsWG) was established, allowing MCST to have more frequent interactions with science discipline representatives, users, and core sensor and calibration experts. In flight, the on-board calibrators are operated to track changes in sensor spectral, spatial, and radiometric properties and to derive calibration parameters used in L1B calibration look-up-table (LUT) updates.

To satisfy MODIS RSB calibration requirements, the SD and SDSM operations are scheduled on a regular basis. For the TEB calibration, the BB is nominally controlled at a fixed temperature. Periodic BB warm-up and cool-down (WUCD) operations are performed, allowing its temperature to vary from instrument ambient to 315K. The SRCA can operate in three different modes: radiometric, spatial, and spectral. Depending on their calibration and characterization purposes, different SRCA modes are used at different frequencies. Other on-orbit calibration and characterization activities include regular lunar observations and electronic calibration (ECAL). Table 1 is a summary of these activities for both Terra and Aqua MODIS. As expected, on-orbit calibration frequencies for SD/SDSM and SRCA operations have gradually decreased as the mission continues. On the other hand, gradually increased efforts have been made to track long-term changes in sensor response via carefully selected ground reference targets and inter-comparisons of MODIS (Terra and Aqua) with other sensors [3].

Table 1 Summary of MODIS on-orbit calibration activities (as of May 31, 2012).

\begin{tabular}{|c|c|c|c|c|c|c|c|}
\hline & $\begin{array}{c}\text { SD / } \\
\text { SDSM }\end{array}$ & MOON & $\begin{array}{c}\text { BB } \\
\text { WUCD }\end{array}$ & $\begin{array}{c}\text { SRCA } \\
\text { RAD }\end{array}$ & $\begin{array}{c}\text { SRCA } \\
\text { SPET }\end{array}$ & $\begin{array}{c}\text { SRCA } \\
\text { SPAT }\end{array}$ & ECAL \\
\hline Terra & 342 & 112 & 81 & 147 & 51 & 66 & 63 \\
\hline Aqua & 231 & 91 & 44 & 119 & 30 & 50 & 52 \\
\hline
\end{tabular}

\section{CALIBRATION METHODOLOGIES}

MODIS bands 1-19 and 26 are the reflective solar bands (RSB), covering wavelengths from 0.41 to $2.2 \mu \mathrm{m}$. MODIS RSB radiometric calibration is reflectance based using a solar diffuser. Its earth view top-of-atmosphere (TOA) reflectance factors are derived or calibrated with reference to the SD bi-directional reflectance factor (BRF). RSB calibration coefficients, used in a linear calibration and retrieval algorithm, are derived and updated using SD and SDSM observations. The SD BRF was characterized prelaunch. The SDSM is used to track on-orbit changes in SD BRF with its alternate measurements of the SD and Sun view responses. The RSB detector responses to the SD and earth view (EV) are corrected for the instrument background and scan angle differences. The RSB radiances are converted from the reflectance factors by multiplying the solar spectral irradiance integrated over detectors' relative spectral response (RSR).

For MODIS TEB (bands 20-25 and 27-36) with wavelengths ranging from 3.7 to $14.2 \mu \mathrm{m}$, a quadratic algorithm is applied to derive the calibration coefficients based on $\mathrm{BB}$ measurements and to retrieve the sensor $\mathrm{EV}$ radiances. In BB calibration, thermal emission from the scan mirror and that from the scan cavity (reflected from the BB) are included as source contributions. A scan angle dependent mirror term is also included in the EV radiance retrieval equation. The scan-by-scan calibration at a fixed BB temperature is used only to derive the dominant linear coefficients for the quadratic algorithm. The offset and nonlinear terms used are derived from pre-launch calibration and updated using on-orbit data collected from BB WUCD over a range of $\mathrm{BB}$ temperatures. For the bands that experience either electronic crosstalk or an optical leak, additional correction algorithms are designed and applied to mitigate the impact on both the calibration and retrieval. More details of MODIS calibration methodologies can be found from MCST publications [4, 5].

\section{CALIBRATION RESULTS AND DISCUSSIONS}

Overall MODIS instrument on-orbit operation performance has been very stable. Changes in instrument temperatures have been less than $3.5 \mathrm{~K}$ over 12 years for Terra MODIS and less than $2.5 \mathrm{~K}$ over 10 years for Aqua MODIS. As expected, the MODIS scan cavity, the scan mirror, and the two warm focal plane assemblies (FPA) for the VIS and NIR (near infrared) spectral bands have experienced nearly identical temperature changes. MODIS also has cold FPA (CFPA), one for the SMIR (short- and mid-wave infrared) bands and the other for the LWIR bands. Their temperatures are nominally controlled at $83 \mathrm{~K}$ as shown in Figures 1 and 2. Ignoring expected changes caused by switching between different sensor operational configurations, the CFPA temperatures for Terra MODIS have been very stable. By comparison, Aqua MODIS CFPA temperatures have seen a 
gradual small increase in recent years, coupled with noticeable seasonal variations, due to the loss of its radiative cooler margin. Since launch, Aqua MODIS CFPA temperature has increased about $0.25 \mathrm{~K}$. Not illustrated in Figure 2 are similar but slightly large daily and orbit-to-orbit variations of the CFPA temperatures. Because of this, special efforts have been made in the upcoming MODIS data collection 6 (C6) to minimize calibration impact due to variations of Aqua MODIS CFPA temperatures. Since the TEB calibration is on a scan-by-scan basis, corrections for FPA temperature variations are only needed during BB WUCD when the BB temperatures are above the saturation temperatures for Aqua bands 33, 35, and 36 and when the BB WUCD data sets are used to derive nonlinear calibration coefficients.



Figure 1 Terra MODIS CFPA temperatures (red for SMIR FPA and blue for LWIR FPA)

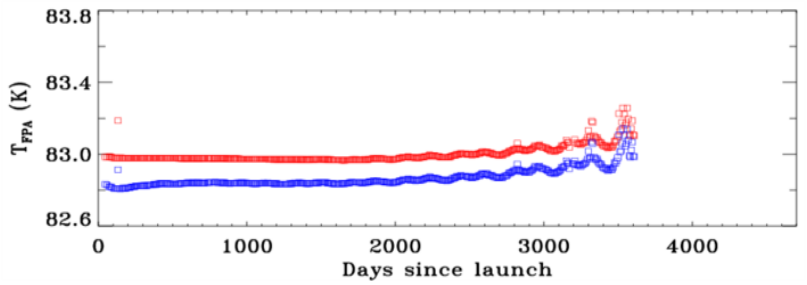

Figure 2 Aqua MODIS CFPA temperatures (red for SMIR FPA and blue for LWIR FPA)

Terra MODIS on-board BB temperatures have been nominally operated at $290 \mathrm{~K}$ and Aqua MODIS at $285 \mathrm{~K}$. Their weekly averaged temperatures are illustrated in Figures 3 and 4 over their entire missions. BB temperatures during BB WUCD are excluded from the above weekly averages. For Terra MODIS, the BB temperature changes are very small in terms of seasonal variations $( \pm 15 \mathrm{mK})$ and long-term drift (30mK). A step-wise increase at around day 300 (since launch) is due to instrument configuration change from A-side to the B-side electronics. By comparison, Aqua MODIS BB performance has been extremely stable with essentially no seasonal variations and long-term drift.

Figures 5 and 6 show on-orbit changes in Terra and Aqua MODIS SD BRF (or SD degradation) determined from SDSM observations. For Terra MODIS, the SD onorbit degradation became much faster after July 2003 (near day 1280 since launch), which corresponds to the time when the SD door was fixed at the "Open" position. Currently, changes in Terra MODIS SD BRF have reached 50\% at $0.412 \mu \mathrm{m}$ (black) , $35 \%$ at $0.465 \mu \mathrm{m}$ (red), and $20 \%$ at 0.533 $\mu \mathrm{m}$ (blue) and changes in Aqua MODIS SD BRF have been approximately $19 \%, 12 \%$, and $7 \%$ at the corresponding wavelengths. As illustrated in Figures 5 and 6, on-orbit changes in SD BRF are strongly wavelength dependent with larger changes occurring at shorter wavelengths.

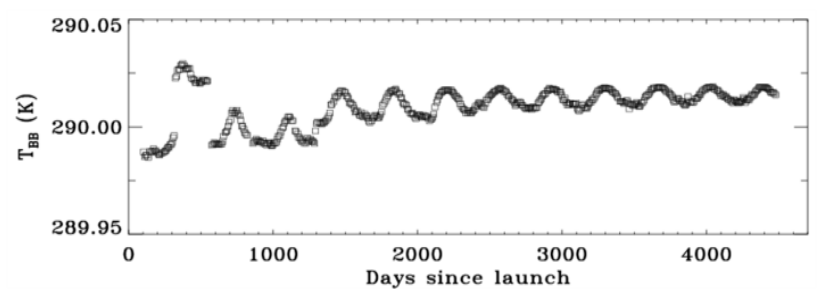

Figure 3 Terra MODIS on-board BB temperatures

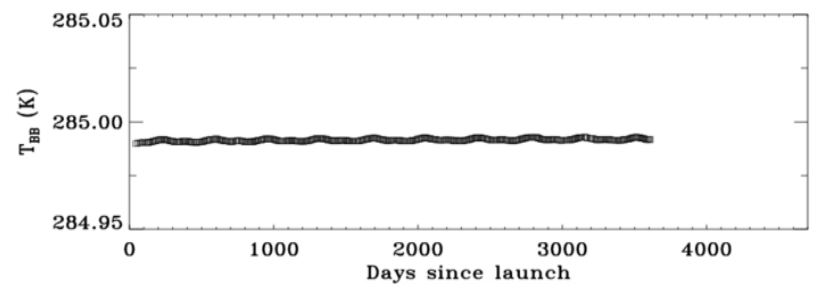

Figure 4 Aqua MODIS on-board BB temperatures

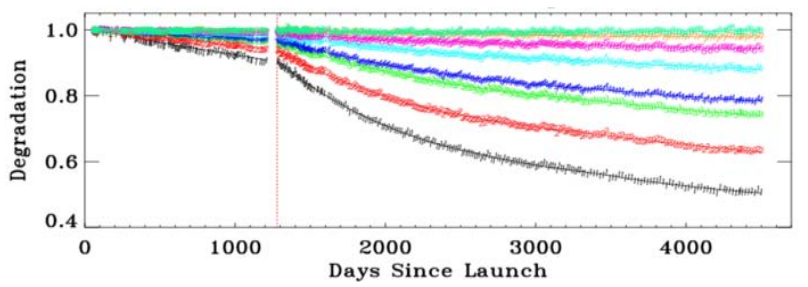

Figure 5 Terra MODIS on-board SD degradation

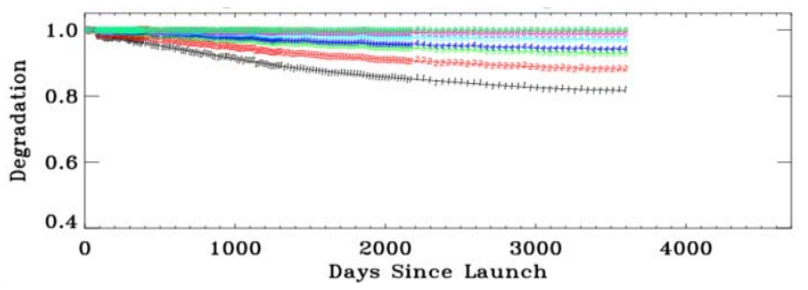

Figure 6 Aqua MODIS on-board SD degradation

For RSB, changes in detector responses (gains) are determined from SD observations after removing the impact due to SD BRF on-orbit degradation and solar viewing geometry difference. For TEB, the BB is used to monitor detector gain changes. In general, the VIS spectral bands at shorter wavelengths have experienced larger changes. This has been the case for both mirror sides and for both Terra and Aqua MODIS. The center wavelengths of MODIS VIS bands are listed in Table 2. Figures 7 and 8 are on-orbit changes in Terra and Aqua MODIS VIS spectral band responses, derived from SD observations from mirror side 1. As expected, on-orbit changes in VIS band responses are also strongly dependent on the AOI to the scan mirror. The 
mirror side differences in Aqua MODIS VIS responses are much smaller than that in Terra MODIS. For both Terra and Aqua MODIS, some NIR bands have experienced an onorbit gain increase. For the SWIR, MWIR, LWIR bands on the CFPA, the changes in their detector responses have been very small. MODIS has a total of 490 individual detectors. After more than 12 years, there are 45 noisy detectors (30 from pre-launch) and no inoperable detectors in Terra MODIS. For Aqua MODIS after 10 years, there are only 7 noisy detectors (2 from pre-launch) and 13 inoperable detectors (10 from pre-launch). In recently years, most detectors' noise characterization performance has been very stable with only one new noisy detector in Aqua MODIS.

Table 2 Specified center wavelengths for MODIS VIS bands.

\begin{tabular}{|c|c|c|c|c|c|c|c|}
\hline VIS bands & $\mathbf{8}$ & $\mathbf{9}$ & $\mathbf{3}$ & $\mathbf{1 0}$ & $\mathbf{1 1}$ & $\mathbf{1 2}$ & $\mathbf{4}$ \\
\hline $\boldsymbol{\lambda}(\mathbf{n m})$ & 412 & 443 & 469 & 488 & 531 & 551 & 555 \\
\hline
\end{tabular}

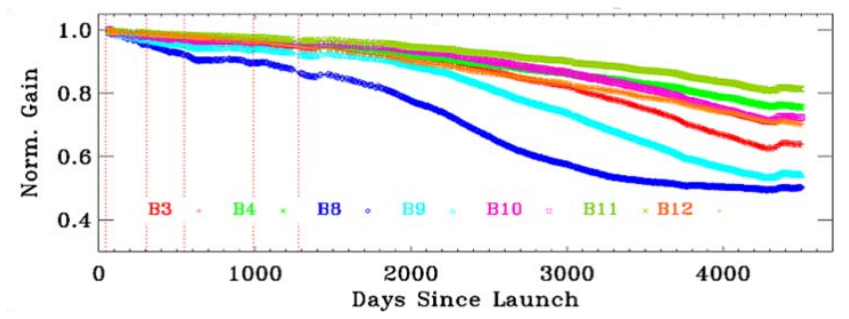

Figure 7 Normalized gains of Terra MODIS VIS bands

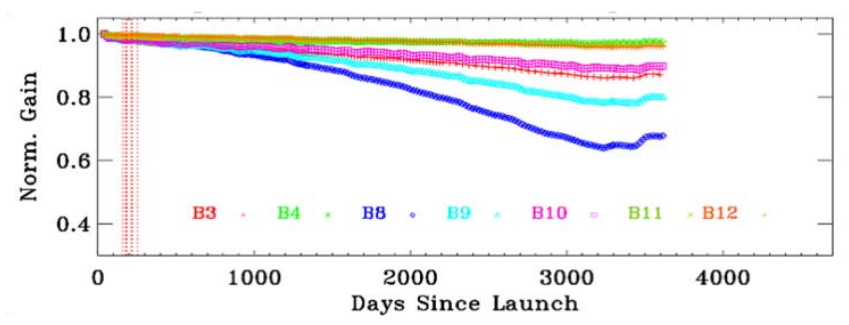

Figure 8 Normalized gains of Aqua MODIS VIS bands

Both instruments have also shown excellent and stable spectral and spatial performance. Typical changes in their center wavelengths (CW) have been less than $0.5 \mathrm{~nm}$ and bandwidths (BW) less than $1.0 \mathrm{~nm}$ for most VIS and NIR bands. Except for the initial band-to-band registration (BBR) offsets identified from pre-launch, there have been little or no on-orbit changes for their along-scan and alongtrack BBR. It is anticipated that, with dedicated calibration efforts, both Terra and Aqua MODIS will continue to provide quality data products and make great contributions to a broad range of scientific studies and applications.

Based on the need by the science and user community and recommendations from the NASA HQ Senior Review Panel, both Terra and Aqua missions have already been extended for several years beyond their 6-year design life time. As a result, some of the calibration frequencies have been gradually reduced. In addition to using SD and lunar observations at fixed AOI, long-term trending of sensor responses at a wide range of AOI over selected ground targets has become increasingly important in support of RVS characterization for the bands with large on-orbit changes. With support from the MODIS program and project, MCST will continue its dedicated efforts to address challenging issues and to maintain sensor on-orbit calibration and data quality in support of a broad science and user community, and to assure mission success for both Terra and Aqua MODIS.

\section{SUMMARY}

Since launch, Terra and Aqua MODIS have made more than 12 and 10 years of continuous observations of the earth's surface and generated various data products in support of a variety of remote sensing research and applications. Onorbit operation and calibration results show that both instruments and their on-board calibrators continue to be able to perform their key design functions and allow calibration and data quality to be maintained. Despite a couple of problems identified pre-launch and a small increase and variation of its CFPA temperatures, Aqua MODIS on-orbit overall performance has continuously been better and more stable than Terra MODIS. Issues identified and resolved during MODIS on-orbit calibration and characterization, and lessons learned through all $\mathrm{Cal} / \mathrm{Val}$ phases will greatly benefit other earth-observing sensors, such the VIIRS on the Soumi NPP mission.

\section{REFERENCES}

[1] V. Salomonson, W. Barnes, X. Xiong, S. Kempler, and E. Masuoka, "An Overview of the Earth Observing System MODIS Instrument and Associated Data Systems Performance," Proc. IEEE IGARSS, doi:10.1109/IGARSS.2002.01025746, pp. 970-972, 2002

[2] X. Xiong, K. Chiang, J. Esposito, B. Guenther, and W.L. Barnes, "MODIS On-orbit Calibration and Characterization,” Metrologia 40, 89-92, 2003

[3] Xiong X, A. Wu, B. Wenny, J. Choi, and A. Angal, "Progress and Lessons from MODIS Calibration Intercomparison Using Ground Test Sites," Canadian Journal of Remote Sensing Special Issue, 36 (5), 540552, doi: 10.5589/m10-082, 2010

[4] X. Xiong, K. Chiang, J. Sun, W. Barnes, B. Guenther, and V. Salomonson, "NASA EOS Terra and Aqua MODIS On-orbit Performance," Adv. Space Res., 43, 413-422, doi:10.1016/j.asr.2008.04.008, 2008

[5] X. Xiong, B.N. Wenny, and W.L. Barnes, "Overview of NASA Earth Observing Systems Terra and Aqua Moderate Resolution Imaging Spectroradiometer Instrument Calibration Algorithms and On-orbit Performance,” J. Appl. Remote Sens., Vol. 3, 032501, doi:10.1117/1.3180864, 2009 